\title{
Development of New Identification Method For Global Group of Controls For Online Coordinated Voltage Control In Active Distribution Networks
}

\author{
Khaled Alzaareer, Member, IEEE, Maarouf Saad, Senior Member, IEEE, Hasan Mehrjerdi, Member, \\ IEEE, Dalal Asber, and Serge Lefebvre
}

\begin{abstract}
The incorporation of distributed generation (DG) units in distribution network voltage control may create operational conflicts with other conventional voltage control devices. Structural changes of networks can also increase the possibility to create control interactions. Control rules-based voltage control methods are not suitable for large scale networks where large number of control choices are available. Similarly, optimization-based voltage control methods are impractical to implement since many control variables have to be used to obtain the optimal solution. Therefore in this paper, a new technique for choosing a global group of the most effective control variables considering the ones with low cost is proposed for voltage regulation in distribution networks. This technique is based on the concept of electrical distances between the voltage control devices and network nodes to derive a correction index (CI). The index represents the level of effectiveness of each control variable with respect to all violated voltages. The index is implemented in two phase algorithms (top-down and bottom-up) to identify the global group of control variables. The proposed technique takes into consideration five aspects: the effectiveness, availability, and cost of the control variables as well as the structural changes of networks and the coordination between control variables to simultaneously eliminate the violation in the voltages. The technique is fast and suitable to be implemented for online voltage control. The proposed method is successfully examined on the modified IEEE 123 distribution system under different scenarios.
\end{abstract}

Index Terms-Voltage Control; Controls Selection; Controls Coordination; Electrical Distances; Active Distribution Systems.

\section{INTRODUCTION}

Voltage control is one of the operational challenges in active distribution networks. The integration of multiple DG units into distribution networks can cause over/under voltage problems as well as operational conflicts with other conventional voltage control devices, such as on load tap changers (OLTC), Voltage Regulators (VRs), and Capacitor Banks (CBs) [1]. These problems can grow as distribution networks meet structural changes [2]. Thus, it is required to operationally update the voltage control devices in active distribution networks.

One of the favorable ways of reducing the adverse impacts of the operational conflicts among DG units and conventional voltage control devices is control coordination. Generally speaking, the coordinated voltage control (CVC) techniques can update the control actions based on control rules or optimization techniques.

A novel method for CVC in active distribution systems is developed in [3]. The method is based on installing remote terminal units at DG units to communicate with each other. A method for CVC in active distribution systems based on control rules algorithm is proposed in [4]. A Dynamic Master/Slave Scheme for voltage control in active distribution networks is presented in [5]. A coordinated method based on power flow sensitivity factor for output control of multiple DG units are detailed in [6]. In [7], an online combined local and remote voltage control in the presence of induction machine-based DG unit is presented. A CVC method for coordination of OLTC, $\mathrm{VR}$, and DG unit is presented in [8]. The method is based on the concept of control zone, line drop compensation, as well as the controllers' parameters. In [9], real-time voltage control for distribution systems employing plug-in electric vehicles, DG, and OLTC is proposed. However, all the above-mentioned studies do not consider the coordination process in case of multiple conventional voltage control devices and multiple DG units. In [10], an online CVC strategy is developed in order to operationally avoid the impact of simultaneous responses of multiple voltage control devices and DG units. However, it does not consider the impacts of non-simultaneous operations of DG units and the conventional devices. To minimize the impact of non-simultaneous operations, the authors in [2] proposed an online CVC by allowing voltage control devices to operate in accordance with a priority scheme. However, control rulesbased CVC methods are not suitable for large networks where a large number of control choices are available or when many various objectives are included in the control. In these cases, defining of control rules is a complex task.

Different techniques for Optimization-based CVC in active distribution networks have been presented in the literature. In [11], a method is developed for voltage regulation in active distribution systems by formulating a large-scale optimization problem and solving it using a genetic algorithm-based solver. A strategy is proposed in [12] for CVC in active distribution networks using a dynamic programming method. In [13], a comprehensive decentralized Volt/VAr control strategy is proposed for coordination of conventional control devices and synchronous machine-based DG units in distribution networks. 
A multiple line drop compensation voltage control technique for under-load tap changer transformers is proposed in [14], where the desired tap positions are obtained by solving an integer optimization problem. In [15], a strategy for short-term scheduling of DG units and conventional voltage control devices is proposed for implementing optimal voltage control in active distribution networks. A mutation fuzzy adaptive particle swarm optimization method for CVC is proposed in [16]. A Trust Region Sequential Quadratic Programming technique for CVC in active distribution networks is presented in [17]. However, by using optimization-based methods, large number of control variables have to be activated to obtain the best solution, which is not suitable for practical systems. Moreover, those works do not fully consider the impacts of structural changes in voltage control.

The wide distribution of controls and the possibility of occurring many voltage violations among the network makes it is hard to provide coordination of the controls while activating only the required number of controls. Moreover, it is not easy to rank the most effective control variables during multi violated voltages while considering a set of aspects. Thus and in order to avoid the problems associated with the two kinds of CVC methods, this work proposes a new method to identify and coordinate a set of the cheaper and most effective control variables (only the required number) for CVC in active distribution networks. The proposed method is capable of ranking the control variables to multi violated voltages (not only one violated voltage) while considering a set of aspects, especially the cost. It is also capable to simultaneously (with no time delay) eliminate all the multi violations with only the most effective controls, low computational efforts and high speed. The proposed method does not aim to find the optimal voltage control, which requires operation of most, if not all, the control variables, but provides a group of the most effective control variables to simultaneously eliminate the violation in voltage, taking into accounts the cost of controls. Thus, the result of this method provides a feasible solution for practical implementation.

The proposed method employs a correction index based on the concept of electrical distances between the voltage control devices and network nodes to rank the controls. The index represents the level of effectiveness of each control variable with respect to the violated voltages. Then, the index is implemented in two phase algorithms (top-down and bottomup) to identify the global group of controls that are the most efficient to simultaneously eliminate the violation in voltages.

The key contributions of this study are:

1. Using optimization-based CVC methods, large number of controls have to be activated to obtain the best solution, which is not suitable for practical systems. Solution infeasibility is one of the main problems for optimizationbased methods. In contrast, the proposed method can guide the network operators to select a global set of the most effective control variables (only the required number) to eliminate the violation in the voltages, which is feasible for practical implementation.

2. Compared with control rules-based CVC methods, the proposed method is suitable for large networks where a large number of control choices exist or when many various objectives are included in the control. Defining of control rules is a complex task in those cases. Moreover, most of the rules-based methods are not capable to simultaneously eliminate the violations due to the time delay associated with conventional controls.

3. The proposed method shows a high level of flexibility such that it can take into consideration the five aspects below to eliminate the violation in the voltages: the effectiveness, availability, and the cost of each control action as well as the structural changes of networks and the coordination among control actions.

4. Since the proposed technique mainly depends on the concept of electric distances, it is able to effectively account for the impact of structural changes in the grid.

The remainder of the paper is organized as follows. Section II shows the calculation of the electrical distances between the control variables and network voltages. Section III formulates the correction index to determine the level of effectiveness of each control variable with respect to the violated voltages. Section IV describes how the most effective control variables are identified using the two-phase algorithm. Section V shows simulation results and Section VI shows the conclusions.

\section{Calculation Of The Electrical Distances}

\section{A. Sensitivities of network voltages to control variables}

OLTC, VRs, CBs, and DG units are the main voltage control devices in modern distribution grids. Under a particular condition, the voltage magnitude at bus $i$ of the network for small changes in control variables can be easily obtained.

For DG units, the sensitivities of network voltages to the power injected by DG units into the network can be found using the inverse Jacobian matrix, $J^{-1}$ as:

$$
\begin{gathered}
{\left[\begin{array}{l}
\Delta \theta \\
\Delta V
\end{array}\right]=\left[J^{-1}\right]\left[\begin{array}{l}
\Delta P \\
\Delta Q
\end{array}\right]} \\
{\left[\begin{array}{l}
\Delta \theta \\
\Delta V
\end{array}\right]=\left[\begin{array}{ll}
\frac{\partial \theta}{\partial P} & \frac{\partial \theta}{\partial Q} \\
\frac{\partial V}{\partial P} & \frac{\partial V}{\partial Q}
\end{array}\right]\left[\begin{array}{l}
\Delta P \\
\Delta Q
\end{array}\right]}
\end{gathered}
$$

Where $\Delta \mathrm{P}$ and $\Delta \mathrm{Q}$ represent the vectors of nodal change in active and reactive power, respectively. $\Delta \theta$ and $\Delta \mathrm{V}$ represent the vectors of nodal change in voltage magnitudes and angles, respectively. $\partial \theta / \partial P$ and $\partial \theta / \partial Q$ are the sensitivity vectors of nodal voltage angle to real and reactive power injection, respectively. $\partial V / \partial P$ and $\partial V / \partial Q$ are the sensitivity vectors of nodal voltage magnitude to real and reactive power injection, respectively.

Let us assume that a DG unit is connected at $\mathrm{x}^{\text {th }}$ node for the distribution network with $\mathrm{n}$ number of nodes. Thus, the change in voltage magnitudes at network nodes due to the output variation of DG unit at $\mathrm{x}^{\text {th }}$ node can be found as:

$$
\begin{aligned}
{\left[\Delta V_{1}, \ldots \Delta V_{i}, \ldots, \Delta V_{x}, \ldots, \Delta V_{n}\right]^{T} } & \\
& =\left[\frac{\partial V_{1}}{\partial P_{x}}, \ldots, \frac{\partial V_{i}}{\partial P_{x}}, \ldots, \frac{\partial V_{x}}{\partial P_{x}}, \ldots, \frac{\partial V_{n}}{\partial P_{x}}\right]^{T} \Delta P_{x} \\
+ & {\left[\frac{\partial V_{1}}{\partial Q_{x}}, \ldots, \frac{\partial V_{i}}{\partial Q_{x}}, \ldots, \frac{\partial V_{x}}{\partial Q_{x}}, \ldots, \frac{\partial V_{n}}{\partial Q_{x}}\right]^{T} \Delta Q_{x} }
\end{aligned}
$$

Where ' $i$ ' denotes the $i^{\text {th }}$ node and ' $n$ ' represents number of network buses. Simplify, for the $\mathrm{i}^{\text {th }}$ node: 


$$
\Delta V_{i}=\frac{\partial V_{i}}{\partial P_{x}} \Delta P_{x}+\frac{\partial V_{i}}{\partial Q_{x}} \Delta Q_{x}
$$

The term associated with active power injected by a DG unit, $\left(\partial V_{i} / \partial P_{x}\right) \Delta P_{x}$, depends on the type of DG unit and its operation. However, the term associated with reactive power injection, $\left(\partial V_{i} / \partial Q_{x}\right) \Delta Q_{x}$ can be used for voltage control. Thus, the value of $\partial V_{i} / \partial Q_{x}$ is used to obtain sensitivity of $\mathrm{i}^{\text {th }}$ bus voltage with respect to the DG unit connected at $x^{\text {th }}$ node.

Remark: In some overvoltage cases (i.e. in cases where reactive power output by DG units does not have sufficient capacity for voltage correction), the output active power can also be used for voltage control. Thus, the term $\left(\partial V_{i} / \partial P_{x}\right)$ is used to obtain sensitivity of $i^{\text {th }}$ bus voltage with respect to the active power output by DG unit connected at $\mathrm{x}^{\text {th }}$ node.

To find the sensitivity of $i^{\text {th }}$ bus voltage with respect to conventional control variables, it is necessary to formulate the power flow equations of the network at fixed operating point (i.e. at the current operating point) as follows:

$$
H(V, u)=0
$$

Where $H$ is the set of equations for reactive power injected at all PQ buses. $V$ and $u$ represent the vectors of bus voltages and control variables, respectively.

By taking the derivative of (4), we obtain:

$$
H_{V} d V+H_{u} d u=0
$$

Where $H_{V}$ and $H_{u}$ are the partial derivatives of the injected reactive power with respect to the voltages and control variables, respectively. Based on (5), the sensitivities of network voltages with respect to a conventional control variable $u_{j}$ can be found as:

$$
\frac{\partial V}{\partial u_{j}}=-\left[H_{V}\right]^{-1} H_{u_{j}}
$$

The term $\left[H_{V}\right]^{-1}$ can be directly found using the inverse of Jacobian matrix. $H_{u_{j}}$ is a known vector and represents how the injected reactive power changes with varying the control variable $u_{j}$ (i.e. $\Delta u_{j}$ ). Thus, the change in network voltages due to the variation in the control variables can be found as:

$$
[\Delta V]=\left[\frac{\partial V}{\partial u}\right][\Delta u]
$$

Let us assume that the voltage control device is connected at $\mathrm{x}^{\text {th }}$ node for the distribution system with $\mathrm{n}$ number of nodes. Thus, the change in the voltages due to the variation in the control variable located at $\mathrm{x}^{\text {th }}$ node can be found as:

$$
\begin{aligned}
{\left[\Delta V_{1}, \ldots, \Delta V_{i}, \ldots, \Delta V_{x}, \ldots, \Delta V_{n}\right]^{T}=} & \\
& {\left[\frac{\partial V_{1}}{\partial u_{x}}, \ldots \frac{\partial V_{i}}{\partial u_{x}}, \ldots \frac{\partial V_{x}}{\partial u_{x}}, \ldots, \frac{\partial V_{n}}{\partial u_{x}}\right]^{T} \Delta u_{x} }
\end{aligned}
$$

Where ' $i$ ' denotes the $i^{\text {th }}$ node and ' $n$ ' represents number of network buses. Simplify, for the $\mathrm{i}^{\text {th }}$ node:

$$
\Delta V_{i}=\frac{\partial V_{i}}{\partial u_{x}} \Delta u_{x}
$$

Thus, the value of $\partial V_{i} / \partial u_{x}$ is used to the obtain sensitivity of $\mathrm{i}^{\text {th }}$ bus voltage with respect to the conventional controls (i.e. CBs, and VRs).

\section{B. Determination of the electrical distances}

The calculation of the electrical distances between network buses depends on the relative variation in voltage magnitudes between two nodes due to a change in reactive power injection at one of the nodes. In other words, the coupling between any two buses can be found by the maximum attenuation of the voltage variation between the two buses as follows [20]:

$$
\Delta V_{i}=\alpha_{i j} \Delta V_{j}
$$

Where $\alpha_{i j}$ represents the attenuation between the two nodes $i$ and $j$. The respective attenuation between any control variable device located at $\mathrm{x}^{\text {th }}$ and any node $\mathrm{i}$ in the network can be derived by dividing the elements of the voltage sensitivity vector stated in (2) or (8) by the $\partial V_{x} / \partial Q_{x}$ or $\partial V_{x} / \partial u_{x}$ as:

$$
\begin{aligned}
\alpha_{i x}=\frac{\partial V_{i}}{\partial Q_{x}} / \frac{\partial V_{x}}{\partial Q_{x}} & \text { for } D G \text { units } \\
\alpha_{i x}=\frac{\partial V_{i}}{\partial u_{x}} / \frac{\partial V_{x}}{\partial u_{x}} & \text { for convenional controls }
\end{aligned}
$$

It is clear from (11) that any change in reactive power injection (i.e. $\Delta \mathrm{Q}$ ) at bus $\mathrm{x}$ could cause a change in the voltages at buses $\mathrm{x}$ and i by $\Delta V_{x}$ and $\Delta V_{i}$, respectively. The change in the voltage at a corresponding node due to reactive power injection at its own bus is greater than power injection at other nodes. This means that $\alpha_{i x}$ has a numerical value between 0 and 1 .

The electrical distance between any control variable located at $\mathrm{x}^{\text {th }}$ node and any node in the network $D_{i x}$ can be derived by:

$$
D_{i x}=-\log \left(\alpha_{i x} \cdot \alpha_{x i}\right)
$$

The normalized electrical distance $d_{i x}$ can be given by:

$$
d_{i x}=\frac{D_{i x}}{\max \left(D_{1 x}, D_{2 x}, \ldots, D_{n x}\right)}
$$

In this section, the electrical distances between voltage control devices and network nodes (i.e. sensitivities of the network voltages to voltage controls) are calculated. The distances can be used to sort the promising control variables for any violated voltage, but there is no coordination between these control variables. The coordination is necessary since each control variable has an effect on more than one node voltage. In the next section, these electrical distances will be implemented to develop an index that sorts and chooses the most efficient controls to bring back the violated voltages within an acceptable range.

\section{FORMULATION OF THE CORRECTIVE INDEX}

In order to obtain the global group of most effective control devices to simultaneously eliminate all violated voltages, this section introduces a new index (correction Index) to measure the effectiveness of each control variable to eliminate all the violations in network voltages. The index is based on the critical distances (i.e. sensitivities) between each control variable and network nodes.

In the previous section, we showed that $\mathrm{d}_{i \mathrm{x}}$ illustrated in (13) represents the normalization of the electrical distance between the control variable device located at $\mathrm{x}^{\text {th }}$ node and any node in the network. The normalized electrical distance equation (13) considers the effectiveness of each control.

The availability of the control can also be taken into account by using a parameterization for the control variables such that $u_{x}=0,1$ or -1 represents the present value, the maximum value or the minimum value of the control variable. However, the previous formula does not include the control cost. 
For each control variable $u_{x}$, let $d_{x}$ be the vector of electrical distances between network nodes and the control variable $u_{x}$ as:

$$
d_{x}=\left[\mathrm{d}_{1 \mathrm{x}}, \mathrm{d}_{2 \mathrm{x}}, \ldots, \mathrm{d}_{\mathrm{ix}}, \ldots, \mathrm{d}_{\mathrm{nx}}\right]
$$

If $C_{\mathrm{x}}$ is considered as the unit cost of the $\mathrm{x}^{\text {th }}$ control variable, the vector $d_{x}$ can be modified as follows:

$$
d_{x}^{\prime}=\frac{d_{\mathrm{x}}}{c_{\mathrm{x}}}
$$

Given a control $u_{x}$, the corrective index CI can be calculated by finding the summation of $d_{i x}^{\prime}$ with respect to all the violated voltages as follows:

$$
C I_{x}=\sum_{i=1}^{N} d_{i x}^{\prime}
$$

Where $\mathrm{N}$ is the number of violated voltages and $C I_{x}$ is the index for the control $u_{x}$. This index measures the ability of a control variable to bring all violated voltages within acceptable limits by taking into account its availability, effectiveness, and cost.

A flowchart of the CI calculation is shown in Fig.1

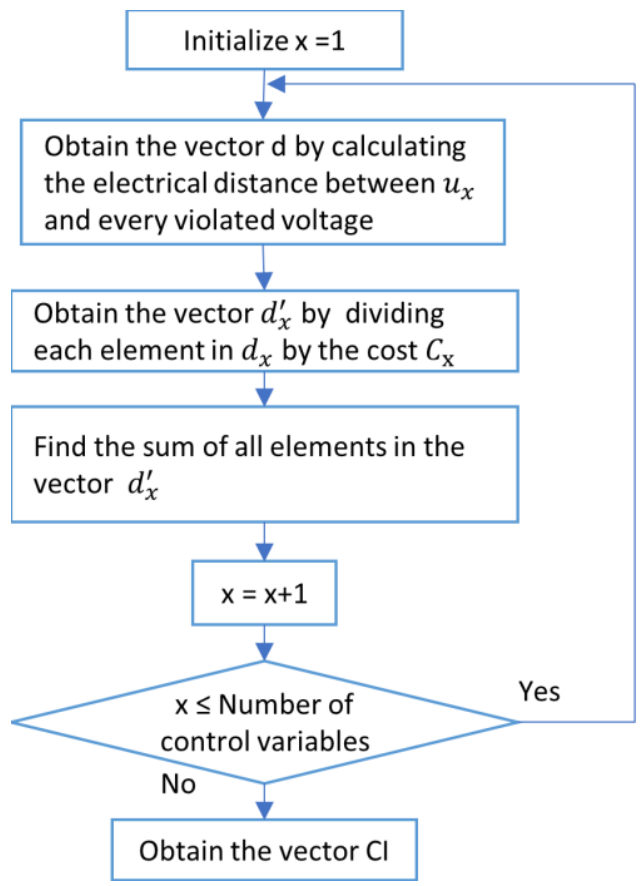

Fig.1: A flowchart of CI calculation

\section{IDENTIFYING Of The Global CONTROL Group}

The development of methods to identify a group of control variables to simultaneously correct the voltages in active distribution networks is necessary. In this section, a two-phase algorithm (top-down and bottom-up) is proposed for this purpose. Top-down and bottom-up are both strategies of data treatment and knowledge organizing, utilized widely in a diversity of fields. The computation effort for the top-down and bottom-up phases is very low and therefore it is suitable for online voltage control.

The basic idea behind the top-down method is that the main task is divided into multiple smaller subtasks. These subtasks are further divided until the obtained subtasks can be simply understood and make it easier to design or implement. Thus, it can be considered as a step-by-step process in which each subtask is separately analyzed for solving the large problem. In contrast, the bottom-up method works in an inverse manner for top-down method. The bottom-up method defines a set of rules for the individual performances and the interactions and then are combined into the entire problem by proceeding with the inference of the full performance. Fig.2 shows the process direction for the two approaches.

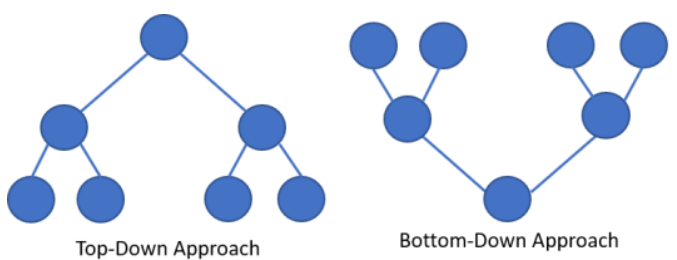

Fig.2: Top-Down approach vs. Bottom-Down approach

Since the subproblems in top-down method aren't connected in a manner so that they can communicate well, redundancies can be obtained. However, the bottom-up approach implements the concept of the information hiding and, thus, it can omit the redundancies. One of the main applications of the bottom-up approach is testing.

In this work, the two approaches have been suggested for identifying the global group of controls for voltage control in active distribution networks. The top-down approach uses aggregate control to obtain the global group, which is then allocated to individual control variables on the basis of their ranking in $\mathrm{CI}$ index. The bottom-up approach employs individual control variables to test the voltage estimation process.

In the first phase (top-down selection), the index vector CI is reordered in descending order, and the control variables (started from the highest rank to the lowest rank) are chosen until all of the violated voltages are eliminated. Thus, this phase tries to choose the control variables with lowest cost and highest effectiveness. By this attempt, the result set from this phase may include excessive controls and consequently increase the overall cost. In the worst case, some voltages may violate the opposite voltage limit. To solve these problems, a second phase (bottom-up selection) is employed.

\section{A. Top-down phase (phase I)}

Let $\Gamma$ be a vector of all violated voltages, i.e., $\Gamma=\left[\mathrm{V}_{1}\right.$, $\left.\mathrm{V}_{2}, \ldots \ldots, \mathrm{V}_{\mathrm{N}}\right]$ and $\varphi$ be the set of the most effective control variables to eliminate the violations in network voltages. Phase I obtains the set $\varphi$ by choosing the control variables with highest CI values. To estimate how much each variable affects the violated voltage, the vector $\Gamma$ is increased by the value of the control variable sensitivity. This selection is performed sequentially until all violated voltages are within the normal voltage limits. Given $\mathrm{N}_{\boldsymbol{c}}$ as the available number of control variable and $\mathrm{x}$ as an index for control variable, the main steps to obtain $\varphi$ during the top-down phase can be explained in the flowchart shown in Fig.3.

\section{B. Bottom-up phase (phase II)}

As mentioned before, top-down strategy might contain more control actions than necessary. This may occur when controls with low CI, like repeated taps of VRs for example, are selected 
to complete the control process. This high cost control variable (low CI) may be redundant to other control variables in set $\varphi$.

To refine the obtained set of control variables $\varphi$, phase II evaluates all the control variables in $\varphi$, starting from the ones with the higher cost, and eliminates the unnecessary ones. Thus, this phase ranks the controls in $\varphi$ in ascending order. To estimate how much eliminating each control variable affects the voltages obtained by phase 1 (let $£=\left\{V_{1}, V_{2} \ldots\right\}$ is the vector of the voltages obtained by phase 1 ), the value of its sensitivity is subtracted from $£$. The control variable is considered useless if its removing from $\varphi$ does not reduce the voltages to a value smaller than Vmin. The set $\varphi^{\prime}$ is the result set by this phase. To obtain a better refinement process, phase 2 can also be performed for each control step $\mathrm{k}$ of control variables in the obtained set $\varphi^{\prime}$ starting from the higher step to the lower step. $\varphi^{\prime \prime}$ is the final result set of the most effective controls with low cost.

Given $\mathrm{N}^{\prime}{ }_{c} \mathrm{~N}^{\prime \prime}{ }_{c}$ as the available numbers of control variables in the group $\varphi$ and $\varphi^{\prime}$ respectively, and $\mathrm{x}$ as an index for control variable in the set $\varphi$, the main steps to refine $\varphi$ during the phase II can be explained in the flowchart shown in Fig.4.

Remark: the previous steps for the two-phase algorithm takes into account only the undervoltage cases. However, the steps can be modified such that the proposed method considers overvoltage cases. This can be achieved by comparing the most violated voltage with the upper acceptable voltage limit Vmax in phase I. Since the availability takes a negative sign during overvoltage cases (i.e. the term $\partial \mathrm{V}_{i} / \partial u_{x}$ ), the new value of violated voltages in the voltage evaluation process will definitely be reduced (instead of increased) by their sensitivities. Similarly, during phase II, the most violated voltage will be compared with Vmax.

\section{Implementation of the proposed voltage control scheme}

Fig. 5 offers an overview of the proposed global voltage control method. It is proposed that the control scheme is implemented using a distribution management system (DMS) for online voltage control. DMS uses pseudo measurements of load and DG generation, and information on system structural changes as inputs. Pseudo measurements can be replaced by installing monitoring devices at the corresponding buses. To transfer the updated information about the status of set-points reference value for DG units and the status of conventional voltage control devices to the control center, DG units and conventional voltage control devices are also proposed to be integrated with a supervisory control and data acquisition (SCADA) system.

Since system reconfiguration can be done online, the structural changes can easily be obtained. If there are no structural changes in the network, the previous values of electric distance are used in the analysis.

\section{Test System AND Simulation Results}

To check the validity of the proposed identification technique in coordinated voltage control, the modified IEEE 123-bus distribution network has been used for simulation. The system and the proposed voltage control approach were simulated in OpenDSS through MATLAB environment. Several scenarios are considered to evaluate the validity of the proposed algorithm in choosing the global group of controls.

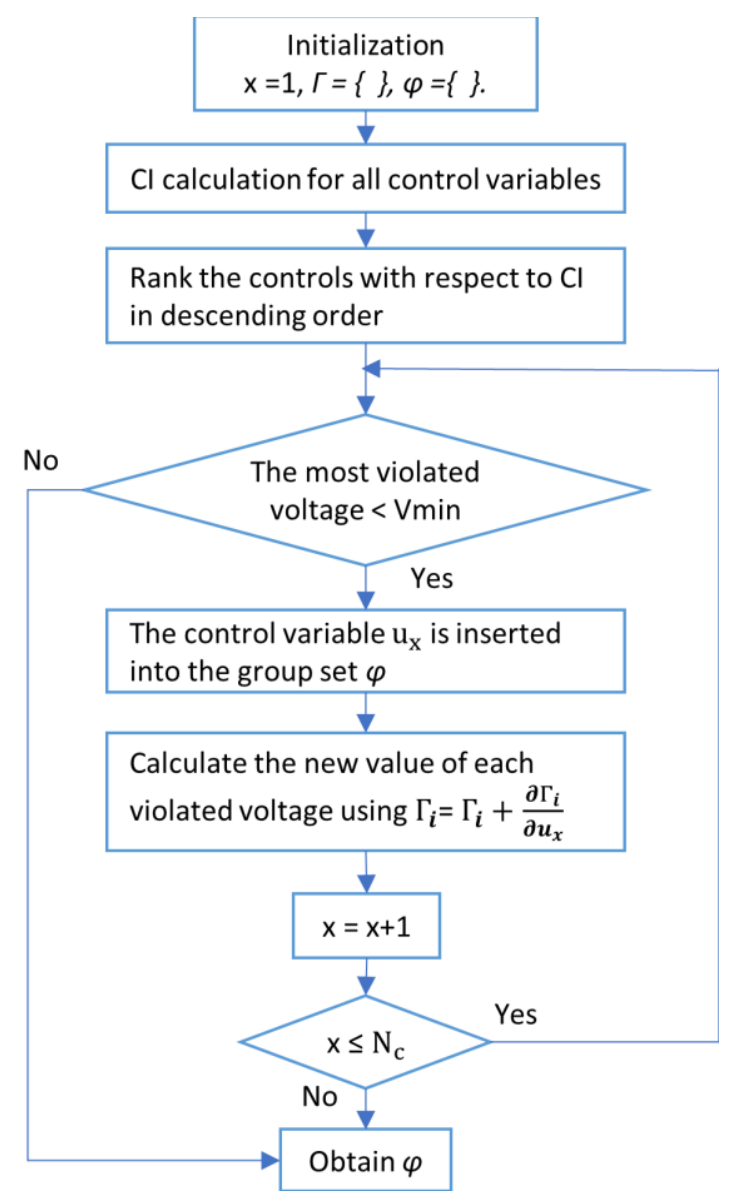

Fig.3: A flowchart of the Top-Down phase of the proposed method
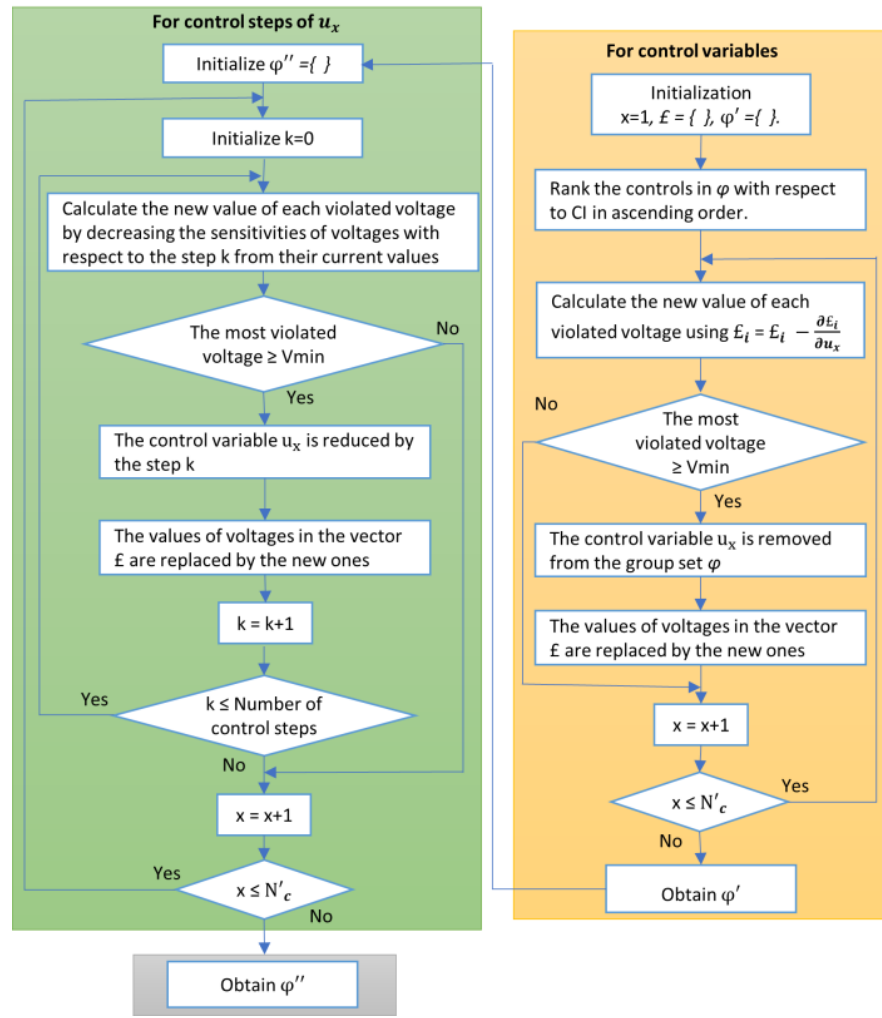

Fig.4: A flowchart of the bottom-up phase of the proposed method 


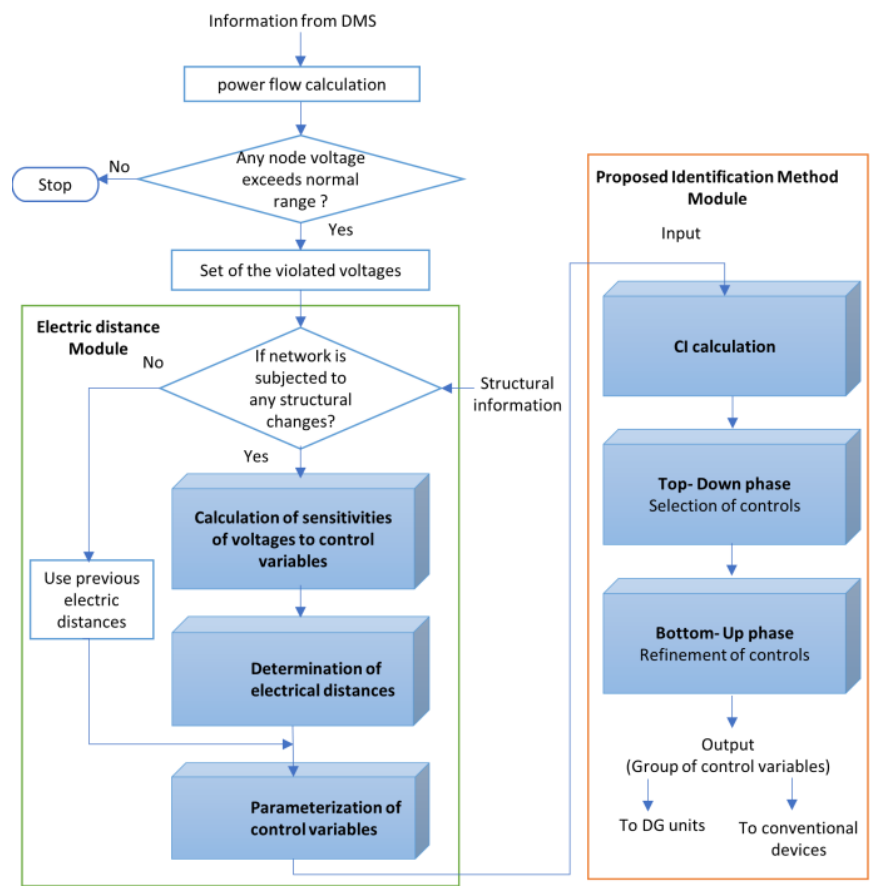

Fig. 5. An overview of the proposed global voltage control

The IEEE 123 distribution network was designed as a multiphase unbalanced system [21]. In this work, the network is modified to be a three-phase balanced grid as the study in [22]. Fig. 6 represents the one-line diagram of the study system. The normal voltage limits of this system is $[0.95,1.03]$ p.u. In the modified IEEE 123-bus system, there are three VRs and four CBs. Ten DG units (each with a capability of $400 \mathrm{KW}$ and 250KVAR) are also installed at 10 buses.

In this work, the output power by DG units, CBs and VRs were considered available for voltage control. Since time delay for OLTC operation is relatively long, it is not included in the voltage control method proposed in this paper. However, if needed, OLTC can also be included in the proposed method by considering its time delay in the availability aspect.

Actually, the cost values assigned to control variables are directly related to the cost of the device to provide ancillary services. Since the controller can distinguish between the cheap and expensive controls, this paper assumes that the cost of change of reactive power injected by DG units are smaller than the costs of other control variables. This is to obtain a higher voltage support by generating reactive power by DG units. Reduction in tap and switching operations is necessary for reducing their maintenance cost and increase in its lifetime and, thus, a much higher cost could be assigned to them rather than other controls. The cost can be determined by network operators based on the type of control variables. Other factors can also be considered such as the age and the required maintenance of the control variable. In this paper, the cost values are chosen by taking into account the type of control variable. The cost values reflect the relative cost of each control. This work also assumes that there are some differences in the cost for each type of control variables. This just to show ability of the proposed method to distinguish between the controls belonging to the same type.

The availability and cost of the different types of control variables are presented in Table I. Shunt capacitor $C_{90}$ and DG

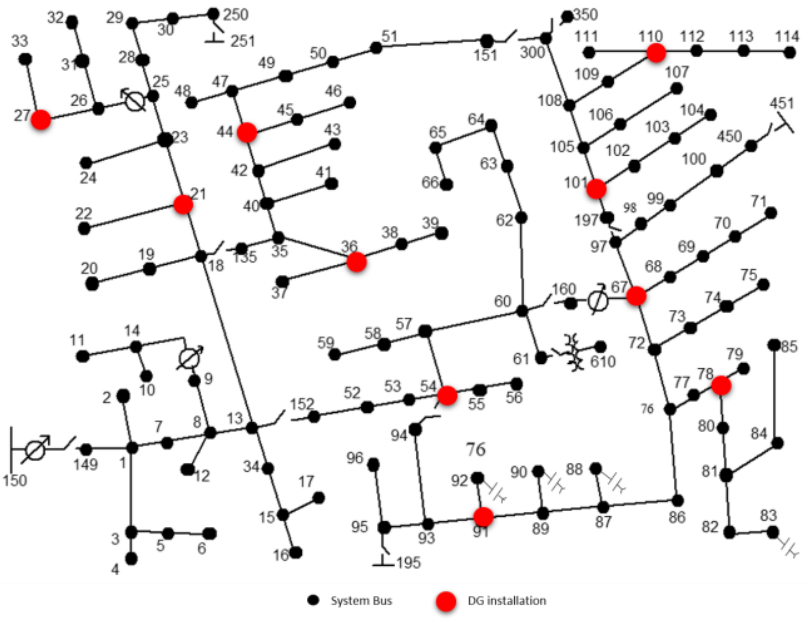

Fig. 6. The modified IEEE 123-bus system

TABLE I

AvAILABILITY AND COST OF CONTROLS

\begin{tabular}{|c|c|c|c|c|c|c|}
\hline \multicolumn{4}{|c|}{ Shunt capacitors } & \multicolumn{3}{|c|}{ Voltage Regulators } \\
\hline $\begin{array}{l}\text { Bus } \\
\text { No. }\end{array}$ & KVAR & Availability & $\begin{array}{l}\text { Cost } \\
\text { (\$) }\end{array}$ & Location & Availability & $\begin{array}{l}\text { Cost } \\
(\$)\end{array}$ \\
\hline 83 & 600 & $75 \%$ & 120 & $9-14$ & $100 \%$ & 1000 \\
\hline 88 & 50 & $50 \%$ & 90 & $25-26$ & $100 \%$ & 1000 \\
\hline 90 & 50 & 0 & 90 & $160-67$ & $100 \%$ & 1000 \\
\hline 92 & 50 & $100 \%$ & 90 & & & \\
\hline
\end{tabular}

\begin{tabular}{|l|l|l|l|}
\hline DG units & \multicolumn{3}{|l|}{} \\
\cline { 1 - 2 } DG location & $\begin{array}{l}\text { Capability } \\
\text { (KVAR) }\end{array}$ & Availability & Cost (\$) \\
\hline 21 & 250 & $10 \%$ & 23 \\
\hline 27 & 250 & $50 \%$ & 25 \\
\hline 36 & 250 & $50 \%$ & 25 \\
\hline 44 & 250 & $75 \%$ & 25 \\
\hline 54 & 250 & $50 \%$ & 20 \\
\hline 67 & 250 & $100 \%$ & 25 \\
\hline 78 & 250 & $0 \%$ & 20 \\
\hline 91 & 250 & $75 \%$ & 23 \\
\hline 101 & 250 & $100 \%$ & 20 \\
\hline 110 & 250 & $100 \%$ & 23 \\
\hline
\end{tabular}

unit $D G_{78}$ are operated at their maximum capacity, i.e., $C_{90}$ $=D G_{78}=1(100 \%)$. Thus, these controls are not available for control.

\section{A. Undervoltage scenario}

\section{Case 1:}

In this case, one operating point is chosen as the representative scenario to examine the validity of the proposed technique in selection the control groups. The system operates at high demand and low power generation by DG units. This results in low voltages at some nodes. After computing the electrical distances between control variables and the violated nodes, the proposed method for selection of control variables was employed to obtain the set of the most effective control variables considering the ones with low cost to eliminate the violation in the voltages. The CI values are calculated and presented in Table II in decreasing order. The table shows that CI values of control variables $C_{90}$ and $D G_{78}$ equals to zero. This because the states of both control variables are at their maximum capacity and therefore there is no available control actions to activate.

From CI calculations, it is also clear that most DG units occupy higher ranks while VRs are at low ranks. This is the 
philosophy of the proposed technique to give the priority for DG units for voltage support. This was achieved by including the cost aspect in the analysis.

The high CI value in Table II represents the most effective control with low cost. Top down phase sequentially selects the control variables from Table II to obtain the lower voltage level (i.e. $\operatorname{Vmin}=0.95$ p.u) . Bottom-up phase enhances the solution of phase 1 by removing unnecessary control variables. The obtained set of control variables is shown in Table III. It is clear that phase I chooses the first seven control variables of Table II with a total cost of $161 \$$. Phase II tried to refine the solution by eliminating the useless control actions, but no one is detected.

If the cost aspect was neglected from CI calculation, the global group of control variables would be $V R_{160}, V R_{25}$, $D G_{101}, D G_{110}, D G_{67}, C_{83}, D G_{91}$, and $V R_{9}$ with a total cost of $3211 \$$. This difference in the cost illustrates the necessary for including the cost aspect in the analysis.

The obtained voltage profiles under three conditions: without control, control using the proposed method and control using an optimization problem (with an objective function to minimize the changes in the control variables) are shown in Fig.7. It is clear that the proposed method is successfully able to mitigate the violation in voltages.

In this case, the results obtained by phase II match the ones obtained by phase I. This means that there is no redundant in the control variables of the set $\varphi$. In other words, any elimination of controls obtained by phase I would cause a violation in the lower level of normal limits (i.e 0.95 p.u).

TABLE II

CI VALUES OF UNDERVOLTAGE SCENARIO

\begin{tabular}{|c|l|c|l|c|l|}
\hline Control No & CI & Control No & CI & Control No & CI \\
\hline$D G_{101}$ & 0.0196 & $D G_{27}$ & 0.0063 & $D G_{21}$ & 0.00085 \\
\hline$D G_{110}$ & 0.0154 & $D G_{36}$ & 0.0058 & $V R_{25}$ & 0.00058 \\
\hline$D G_{67}$ & 0.0144 & $C_{83}$ & 0.0043 & $V R_{9}$ & 0.00028 \\
\hline$D G_{91}$ & 0.0124 & $C_{92}$ & 0.0025 & $D G_{78}$ & 0.0 \\
\hline$D G_{54}$ & 0.0100 & $V R_{160}$ & 0.0022 & $C_{90}$ & 0.0 \\
\hline$D G_{44}$ & 0.0096 & $C_{88}$ & 0.0012 & & \\
\hline
\end{tabular}

TABLE III

Group Of The Controls ObTained Via The Two Phases Of CASE 1

\begin{tabular}{|l|c|c|}
\hline & Top-Down (Phase I) & Bottom-Up (Phase II) \\
\hline $\begin{array}{l}\text { Group of } \\
\text { Controls }\end{array}$ & $D G_{101}, D G_{110}, D G_{67}$, & $D G_{101}, D G_{110}, D G_{67}$, \\
& $D G_{91}, D G_{54}, D G_{44}, D G_{27}$ & $D G_{91}, D G_{54}, D G_{44}, D G_{27}$ \\
\hline Cost & $161 \$$ & $161 \$$ \\
\hline
\end{tabular}

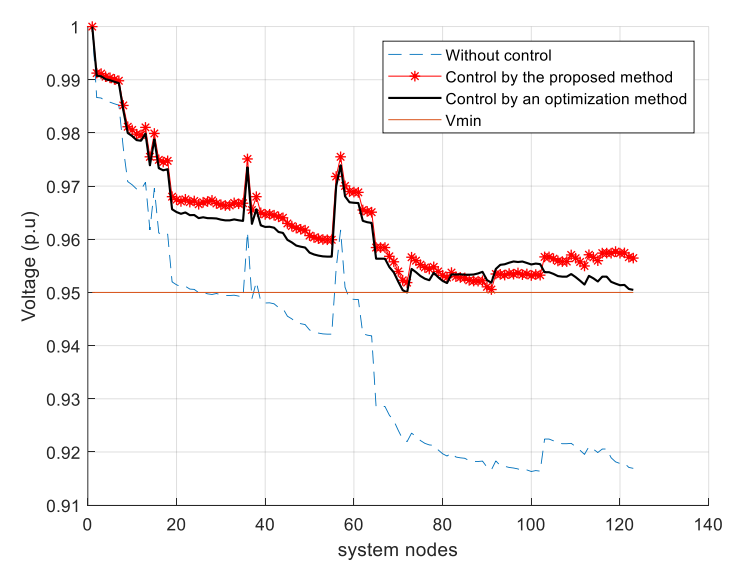

Fig.7. A comparison between the estimated voltage profile obtained by the proposed method and the profiles obtained under other conditions of case 1.
It is worth mentioning that it was found from the results that most of control variables are used for voltage control through the optimization method. In contrast, only the required controls are used for voltage control during the proposed method. Moreover, it was noticed that the control variables obtained by the proposed method have to participate more than other controls during the optimization method. This validates the accuracy of $\mathrm{CI}$ index in selecting the most effective controls. It was also noticed that the control variable with the highest rank have to participate more than other selected controls, which proves the effectiveness of the index CI.

\section{Case 2:}

To show the validity of bottom-up phase in the refinement process, the system is operated at a more severe condition than the previous one. The obtained set of control variables is shown in Table IV. It is clear that phase I chooses the first eleven control variables of Table II with a total cost of 1396 \$. Phase II eliminates one control variable, reducing the cost $1306 \$$. To achieve a better refinement, phase II is also performed for step actions of each control variable of the obtained set. The results showed that the bottom-up phase was able to eliminate 14 taps of the control variable $V R_{160}$, reducing the total cost to $431 \$$ (assuming that the cost for each tap $=1000 / 16 \$$ ).

Table V compares some of the violated voltages with the new estimated values obtained by the two phases. It is clear that the voltages obtained by the phase I is higher than or equal to the ones obtained by the phase II. This due to the fact that the top-down phase overestimates the control requests to get the lower voltage level. From the results, it is also shown that the voltage at bus 102 is the most violated one. The top-down phase chose many control actions until the most violated voltage was larger than Vmin. This phase tried to mitigate the violation in voltages by selecting controls of low cost such as the reactive power output by DG units. However, the DG units have no enough ability to solve the problem. Controls with higher cost are then included in the group to solve the problem. The bottomup phase refined the solution obtained by phase 1 by removing the useless actions while maintaining the most violated voltage greater than Vmin.

The comparison between the estimated voltage profile obtained by the two phases and the profiles under other conditions are shown in Fig.8. It is clear that the proposed method is successfully able to mitigate the violation in voltages. We can also see that the voltages obtained by phase I is higher than or equal to the ones obtained by phase II.

TABLE IV

Group Of The Controls Obtained Via The Two Phases Of CAse 2

\begin{tabular}{|c|c|c|}
\hline & Top-Down (Phase I) & Bottom-Up (Phase II) \\
\hline $\begin{array}{l}\text { Group of } \\
\text { Controls }\end{array}$ & $\begin{array}{c}D G_{101}, D G_{110}, D G_{67}, \\
D G_{91}, D G_{54}, D G_{44}, D G_{27}, \\
D G_{36}, C_{83}, C_{92}, V R_{160}\end{array}$ & $\begin{array}{c}D G_{101}, D G_{110}, D G_{67}, \\
D G_{91}, D G_{54}, D G_{44}, D G_{27}, D G_{36} \\
C_{83}, V R_{160}(\mathrm{tap}=2)\end{array}$ \\
\hline Cost & $1396 \$$ & $431 \$$ \\
\hline
\end{tabular}

Estimated Violated Voltages With The ObTained GRoup Of CASE 2

\begin{tabular}{|l|l|l|l|}
\hline Bus no & Without control & Top-Down & Bottom-Up \\
\hline 13 & 0.9543 & 0.977 & 0.977 \\
\hline 35 & 0.9389 & 0.9589 & 0.9587 \\
\hline 48 & 0.9914 & 0.9525 & 0.9522 \\
\hline 91 & 0.9003 & 1.0352 & 0.9521 \\
\hline 114 & 0.9008 & 1.0362 & 0.9543 \\
\hline
\end{tabular}




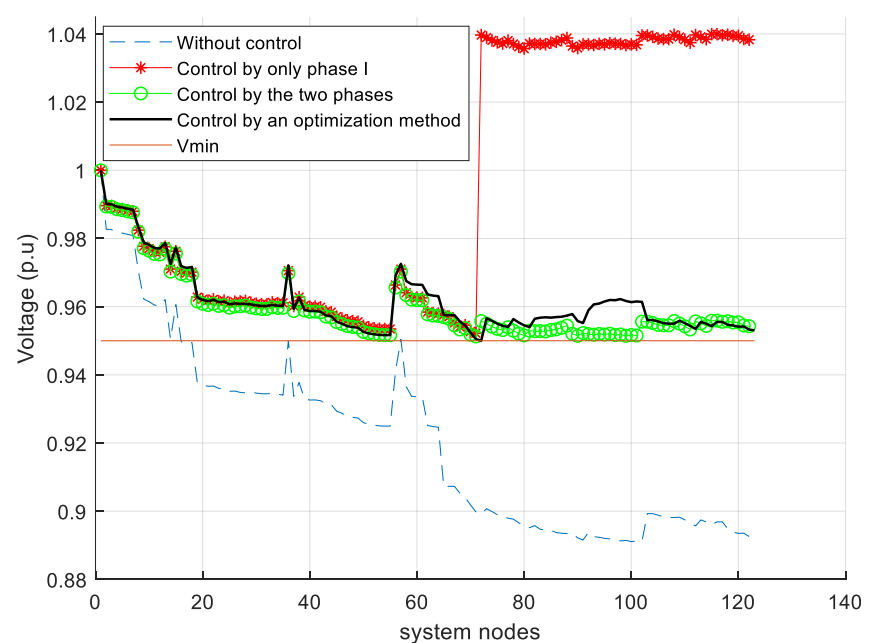

Fig.8. A comparison between the estimated voltage profile obtained by the two phases and the profiles obtained under other conditions of case 2 .

The high difference in the voltages at the end of the curve occurs due to the action of control variable $V R_{160}$. The availability of $V R_{160}$ is $100 \%$ which means that there are 16 taps to enact. During phase I, the control variable $V R_{160}$ with its full availability is chosen for control. However during phase II, the bottom- up phase eliminates the useless control actions of $V R_{160}$ (14 taps in this case). This demonstrates the necessary of the bottom-up phase in the selection process to eliminate the unnecessary actions and, hence, reduce the total cost.

\section{Case 3}

This case shows the performance of the proposed method when most of DG units are installed at the far end of the system. Moreover, this scenario deals with cases where multi DG units are installed at one node or one feeder. Thus, the DG units: $D G_{21}, D G_{27}, D G_{36}, D G_{44}$, and $D G_{54}$ shown in Fig.5 are changed to be placed at the nodes $110,104,71,75$ and 85 respectively (but the numbers assigned with DG units remain unchanged despite of changing their locations). In this scenario, the system also operates at high demand and low power generation by DG units. The obtained set of control variables with the total cost are shown in Table VI. The comparison between the estimated voltage profile obtained by the proposed method and the profiles under other conditions are shown in Fig.9. From the results, it is clear that the proposed method was successfully able to eliminate the violations in the voltage by selecting and refining a global group of controls.

Since most of the voltages are violated at the far end of the system, we can see that all the selected controls are available near their regions. This validates the accuracy of the proposed controller in the selection process. We can also see that the increase amount in the voltages at the region of $0-60$ nodes is less than the amount shown in case 1 . This is due to the fact that more controls are required to be activated in the region of 0-60 nodes of case 1 .

From Table VI we can also see that although two DG units are located at the same node $\left(D G_{21}\right.$ and $\left.D G_{110}\right)$, the controller selects $D G_{110}$ while the unit $D G_{21}$ is ignored. This is due to fact that the proposed method takes into account the availability of control variables in the analysis.
TABLE VI

Group Of The Controls Obtained Via The Two Phases Of Case 3

\begin{tabular}{|l|c|c|}
\hline & Top-Down (Phase I) & Bottom-Up (Phase II) \\
\hline $\begin{array}{l}\text { Group of } \\
\text { Controls }\end{array}$ & $D G_{101}, D G_{110}, D G_{67}$, & $D G_{101}, D G_{110}, D G_{67}$, \\
\hline Cost & $D G_{44}, D G_{91}$ & $D G_{44}, D G_{91}$ \\
\hline
\end{tabular}

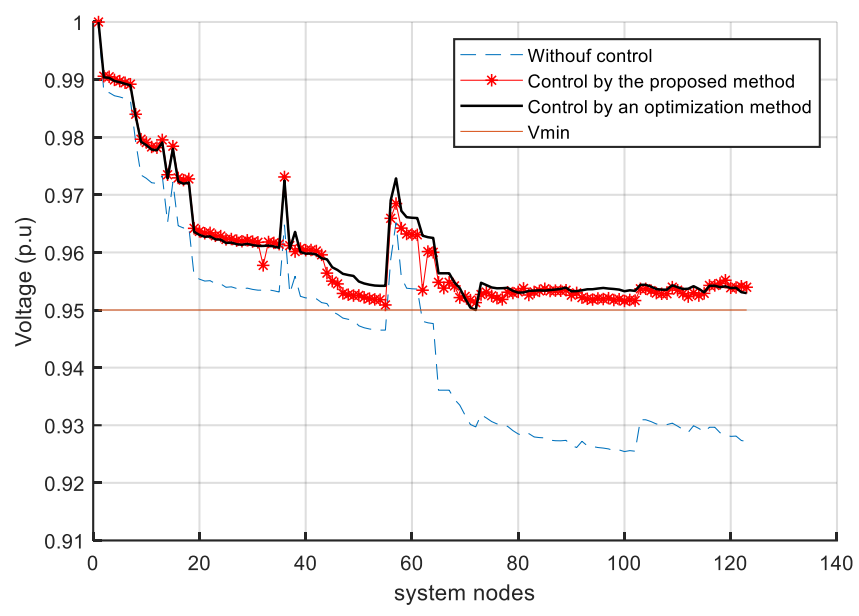

Fig.9. A comparison between the estimated voltage profile obtained by the proposed method and the profiles obtained under other conditions of case 3 .

\section{B. Structural changes scenario}

To check the validity of the proposed technique during structural changes, the first case of undervoltage scenario is repeated but with closing the switch (151-300) and opening the switch (97-197) of the test system. This will make a change in the values of electric distances between nodes. The result of CI values and the group of the most effective controls (during both phases) are presented in Table VII and Table VIII respectively.

It can be seen from table VII that the CI index has a different value and ranking from those presented in scenario 1 . Consequently, we can find that there is a change in the result group of control. The bottom-up phase was able to eliminate four control variables (useless ones), saving a cost of $95 \$$.

Therefore, the proposed method is able to account for the effect of topology changes in the system. This because the proposed algorithm is based on the concept of the electrical distances.

The comparison between the estimated voltage profile obtained by the two phases and the profiles under other conditions are shown in Fig.10. It is clear that the proposed method is successfully able to mitigate the violation in voltages by only the obtained five control variables.

TABLE VII

CI VALUes OF StRuctural CHANGES SCENARIO

\begin{tabular}{|c|l|c|l|c|l|}
\hline Control No & CI & Control No & CI & Control No & CI \\
\hline$D G_{101}$ & 0.0148 & $D G_{27}$ & 0.0063 & $D G_{21}$ & 0.00085 \\
\hline$D G_{67}$ & 0.0144 & $D G_{36}$ & 0.0058 & $V R_{25}$ & 0.00058 \\
\hline$D G_{91}$ & 0.0124 & $C_{83}$ & 0.0043 & $V R_{9}$ & 0.00028 \\
\hline$D G_{110}$ & 0.0116 & $C_{92}$ & 0.0025 & $D G_{78}$ & 0.0 \\
\hline$D G_{54}$ & 0.0100 & $V R_{160}$ & 0.0022 & $C_{90}$ & 0.0 \\
\hline$D G_{44}$ & 0.0096 & $C_{88}$ & 0.0012 & & \\
\hline
\end{tabular}

TABLE VIII

Group Of The Controls ObTained Via The Two Phases Of SCEnARio 2

\begin{tabular}{|l|c|c|}
\hline & Top-Down (Phase I) & Bottom-Up (Phase II) \\
\hline Group of & $D G_{101}, D G_{67}, D G_{91}, D G_{110}$, & $D G_{101}, D G_{67}, D G_{91}$, \\
Controls & $D G_{54}, D G_{44}, D G_{27}, D G_{36}, C_{83}$ & $D G_{110}, C_{83}$ \\
\hline Cost & $276 \$$ & $181 \$$ \\
\hline
\end{tabular}




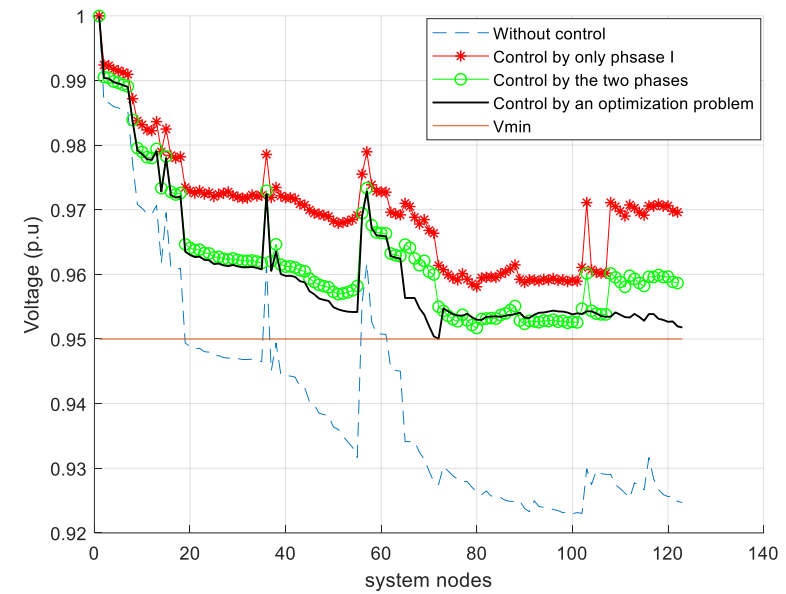

Fig.10. A comparison between the estimated voltage profile obtained by the two phases and the profiles obtained under other conditions for scenario 2.

\section{Overvoltage Scenario}

In this scenario, the system operates at low power demand and high-power generation by DG units. This results in overvoltage at some nodes. For overvoltage cases, active power curtailments can be included as control variables but with a high cost. KW Capability, availability, and cost of DG units are shown in table IVV. The KVAR data presented in Table I are also used for overvoltage control but with an assumption that the availability is in a negative direction (i.e. the KVAR availability is $100 \%$ for $D G_{78}$ and $0 \%$ for $D G_{110}$ ).

The CI values for controls are calculated and ranked. The results showed that most of KVAR controls occupy higher ranks than active power curtailments due to taking into accounts the cost aspect. By performing the proposed method for identifying the global group of control, we found that the units $D G_{78}, D G_{54}, D G_{21}, D G_{27}, D G_{36}$, and $D G_{91}$ are selected for KVAR control while the units $D G_{110}, D G_{67}$ and $D G_{78}$ are selected for active power curtailment in order to eliminate all the violated voltages.

Since the availability for $D G_{110}, D G_{101}, D G_{67}$ for overvoltage control is $0 \%$, they aren't selected in the global group of controls. If the cost was not included in the analysis, we will find that most of DG units will be selected for active power curtailments instead of KVAR control. This is due to the fact that most of DG units have high levels of KW availability compared with KVAR capability.

The comparison between the estimated voltage profile obtained by the proposed method and the profiles under other conditions are shown in Fig.11. It is clear that the proposed method is successfully able to mitigate the violation in the voltages.

TABLE IVV

KW AvaILABILITY AND Cost OF DG UNITS

\begin{tabular}{|l|l|l|l|}
\hline $\begin{array}{l}\text { DG } \\
\text { location }\end{array}$ & $\begin{array}{l}\text { capability } \\
\text { (KW) }\end{array}$ & Availability & Cost (\$) \\
\hline 21 & 350 & $90 \%$ & 300 \\
\hline 27 & 350 & $70 \%$ & 300 \\
\hline 36 & 350 & $80 \%$ & 300 \\
\hline 44 & 350 & $90 \%$ & 300 \\
\hline 54 & 350 & $70 \%$ & 300 \\
\hline 67 & 350 & $90 \%$ & 300 \\
\hline 78 & 350 & $80 \%$ & 300 \\
\hline 91 & 350 & $70 \%$ & 300 \\
\hline 101 & 350 & $70 \%$ & 300 \\
\hline 110 & 350 & $90 \%$ & 300 \\
\hline
\end{tabular}

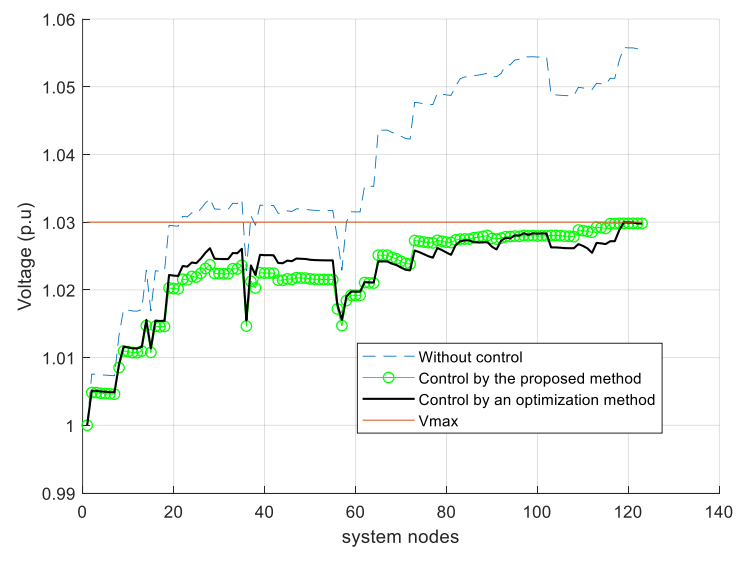

Fig.11. A comparison between the voltage profile obtained by the proposed method and the profiles obtained under other conditions for scenario 3 .

\section{Dynamic Simulation Studies}

The applicability of the proposed technique for online voltage control can be examined through dynamic response. The operating condition presented for the case one of undervoltage scenario is repeated for this scenario. The only change is that all DG units are assumed to be solar PV systems. To get a dynamic response, the test system is operated under rapid solar variations. The solar changes start by declining from $100 \%$ at $\mathrm{t}=0 \mathrm{sec}$ to $0.09 \%$ at $\mathrm{t}=34 \mathrm{ses}$. Then, the changes are stopped for $6 \mathrm{sec}$. After that, the solar changes start to increase to reach $100 \%$ at $t=72 \mathrm{sec}$. The same cycle is repeated until $120 \mathrm{sec}$ is completed.

Remark: The obtained controls in the global set in the corresponding case are all DG units. Thus, in order to show the speed of the proposed method, it is assumed that the control actions will take place during seconds. However, control adjustments can be required every minute, 15 minutes, or every day. This is governed by (a) the time delay associated with conventional controls and (b) the available input data to the controller (i.e. load and DG generation).

The node having the most violated voltage, node 85 , is chosen for dynamic studies. The comparison between the estimated voltages of node 85 obtained by the proposed method and the voltages with no control is presented in Fig. 12. It is clear that the proposed method for voltage control has a very fast response. This is due to the fact that the proposed method has a very simple calculation.

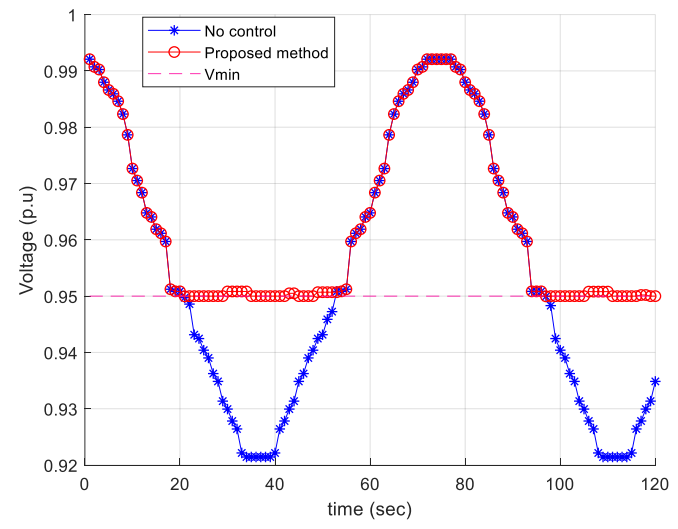

Fig. 12. The comparison between the estimated voltages of node 85 obtained by the proposed method and the voltages with no control. 


\section{CONCLUSIONS}

In this work, a new identification method to determine a global group of controls for online $\mathrm{CVC}$ for active distribution systems is developed. Only the most effective control variables with the low-cost ones are identified as a global group to simultaneously bring back all the violated voltages inside the normal voltage limits. The proposed technique takes into the consideration the effectiveness, availability, and cost of the control variables as well as the structural changes of networks and the coordination between control variables to simultaneously correct all violated voltages. This method depends on the concept of electrical distances between control variables and system buses. The top-down and bottom-up phases are used to achieve the selection process.

Tests were conducted on the Modified IEEE 123-bus network. The control variables were successfully grouped in terms of their effectiveness and cost to mitigate the violation in the voltages. Activation of the control variables of the obtained group demonstrates that all violated voltages are restored inside the normal limits with a low cost. Thus, the proposed method can guide the network operator to choose the minimum number of controls to eliminate the violations in voltages.

Since the proposed identification algorithm mainly depends on the concept of the electrical distances, the proposed method was effectively able to account for the effect of topology changes in the grid.

The proposed method was also checked in a dynamic simulation. Dynamic results demonstrate the efficacy of the proposed method in voltage control with fast response time.

Further work is required to take into account the OLTC in the proposed voltage considering its time delay and in a dynamic analysis. The transient performance of distribution network under the action of the proposed method and under different scenarios is also part of our vision.

\section{ACKNOWLEDGMENT}

This work was supported by the National Priorities Research Program under Grant 11S-1125-170027 from the Qatar National Research Fund (a member of Qatar Foundation).

\section{REFERENCES}

[1] D. Ranamuka, A. P. Agalgaonkar and K. M. Muttaqi, "Examining the Interactions between DG Units and Voltage Regulating Devices for Effective Voltage Control in Distribution Systems," in IEEE Transactions on Industry Applications, vol. 53, no.2, pp. 1485-1496, March-April 2017.

[2] D. Ranamuka, A. P. Agalgaonkar and K. M. Muttaqi, "Online Coordinated Voltage Control in Distribution Systems Subjected to Structural Changes and DG Availability," in IEEE Transactions on Smart Grid, vol. 7, no. 2, pp. 580-591, March 2016.

[3] M. E. Elkhatib, R. El-Shatshat and M. M. A. Salama, "Novel Coordinated Voltage Control for Smart Distribution Networks With DG," in IEEE Transactions on Smart Grid, vol. 2, no. 4, pp. 598-605, Dec. 2011

[4] A. Kulmala, S. Repo and P. Järventausta, "Coordinated Voltage Control in Distribution Networks Including Several Distributed Energy Resources," in IEEE Transactions on Smart Grid, vol. 5, no. 4, pp. 20102020, July 2014.

[5] M. S. El Moursi, H. H. Zeineldin, J. L. Kirtley and K. Alobeidli, "A Dynamic Master/Slave Reactive Power-Management Scheme for Smart Grids With Distributed Generation," in IEEE Transactions on Power Delivery, vol. 29, no. 3, pp. 1157-1167, June 2014.

[6] S. C. E. Jupe, P. C. Taylor, and A. Michiorri, "Coordinated output control of multiple distributed generation schemes," in IET Renew. Power Gener., vol. 4, no. 3, pp. 283-297, May 2010.
[7] F. A. Viawan and D. Karlsson, "Combined Local and Remote Voltage and Reactive Power Control in the Presence of Induction Machine Distributed Generation," in IEEE Transactions on Power Systems, vol. 22, no. 4, pp. 2003-2012, Nov. 2007.

[8] K. M. Muttaqi, A. D. T. Le, M. Negnevitsky and G. Ledwich, "A Coordinated Voltage Control Approach for Coordination of OLTC, Voltage Regulator, and DG to Regulate Voltage in a Distribution Feeder," in IEEE Transactions on Industry Applications, vol. 51, no. 2, pp. 12391248, March-April 2015.

[9] M. A. Azzouz, M. F. Shaaban and E. F. El-Saadany, "Real-Time Optimal Voltage Regulation for Distribution Networks Incorporating High Penetration of PEVs," in IEEE Transactions on Power Systems, vol. 30, no. 6, pp. 3234-3245, Nov. 2015.

[10] D. Ranamuka, A. P. Agalgaonkar and K. M. Muttaqi, "Online Voltage Control in Distribution Systems With Multiple Voltage Regulating Devices," in IEEE Transactions on Sustainable Energy, vol. 5, no. 2, pp. 617-628, April 2014.

[11] T. Senjyu, Y. Miyazato, A. Yona, N. Urasaki and T. Funabashi, "Optimal Distribution Voltage Control and Coordination With Distributed Generation," in IEEE Transactions on Power Delivery, vol. 23, no. 2, pp. 1236-1242, April 2008.

[12] Y. Kim, S. Ahn, P. Hwang, G. Pyo and S. Moon, "Coordinated Control of a DG and Voltage Control Devices Using a Dynamic Programming Algorithm," in IEEE Transactions on Power Systems, vol. 28, no. 1, pp. 42-51, Feb. 2013.

[13] F. A. Viawan and D. Karlsson, "Voltage and Reactive Power Control in Systems With Synchronous Machine-Based Distributed Generation," in IEEE Transactions on Power Delivery, vol. 23, no. 2, pp. 1079-1087, April 2008.

[14] J.-H. Choi and J.-C. Kim, "Advanced voltage regulation method of power distribution systems interconnected with dispersed storage and generation systems," in IEEE Transactions on Power Delivery, vol. 16, no. 2, pp. 329-334, April 2001.

[15] A. Borghetti et al., "Short-Term Scheduling and Control of Active Distribution Systems With High Penetration of Renewable Resources," in IEEE Systems Journal, vol. 4, no. 3, pp. 313-322, Sept. 2010.

[16] H. Yang and J. Liao, "MF-APSO-Based Multiobjective Optimization for PV System Reactive Power Regulation," in IEEE Transactions on Sustainable Energy, vol. 6, no. 4, pp. 1346-1355, Oct. 2015.

[17] W. Sheng, K. Liu, S. Cheng, X. Meng and W. Dai, "A Trust Region SQP Method for Coordinated Voltage Control in Smart Distribution Grid," in IEEE Transactions on Smart Grid, vol. 7, no. 1, pp. 381-391, Jan. 2016.

[18] M. R. Vallem, B. Vyakaranam, J. T. Holzer, M. A. Elizondo and N. A. Samaan, "Power system decomposition for practical implementation of bulk-grid voltage control methods," 2017 19th International Conference on Intelligent System Application to Power Systems (ISAP), San Antonio, TX, pp. 1-6, 2017.

[19] IEEE PES, Distribution Test Feeders, Sep. 2010. [Online]. Available: http://www.ewh.ieee.org/soc/pes/dsacom/testfeeders/index.html.

[20] B. Zhao, Z. Xu, C. Xu, C. Wang and F. Lin, "Network Partition-Based Zonal Voltage Control for Distribution Networks With Distributed PV Systems," in IEEE Transactions on Smart Grid, vol. 9, no. 5, pp. 40874098, Sept. 2018. 